\title{
Detection and analysis of DNA material in human blastocoel fluid
}

\author{
Tao Shangguan ${ }^{1}$, Wei He ${ }^{1 *}$, Hongmei Li ${ }^{1}$, Xiaoyun Shang ${ }^{2}$, Yonggang Liu ${ }^{1}$, Xueyao Bai ${ }^{2}$, Mingduo Li ${ }^{1}$ and Jiafei Xie ${ }^{1}$ \\ ${ }^{1}$ Reproductive Medical Center, Department of Obstetrics and Gynecology, Southwest Hospital, Third Military Medical University, Chongqing, China \\ ${ }^{2}$ Institute of Immunology, Third Military Medical University, Chongqing, China
}

\begin{abstract}
Preimplantation genetic diagnosis (PGD) is becoming a widely-accepted technique during in vitro fertilization (IVF). However, a disadvantage of PGD is the invasive biopsy methods used to sample embryonic cells or polar bodies. Recent studies have found that genetic material can be detected in blastocoel fluid (BF) and culture medium. In our study, BF and trophectoderm (TE) cells were simultaneously collected from the same donated human blastula. To generate enough DNA for analysis, we used multiple displacement amplification (MDA) based whole genome amplification (WGA). MDA-WGA samples were probed with primers designed to identify spinal muscular atrophy (SMA) and phenylpropionate ketoneuria (PKU), plus the Y chromosome sex-determining region (SRY). This demonstrated that DNA fragments were present in each of the TE and BF samples (7/7). The positive PCR amplification rates for SMA, PKU, SRY and $\beta$-actin in BF were $42.9 \%$ $(3 / 7), 60 \%(3 / 5), 42.9 \%(3 / 7)$ and $71.4 \%(5 / 7)$ respectively, but the positive rate of amplification with TE samples was $100 \%(7 / 7), 100 \%(7 / 7), 71.43 \%(5 / 7)$ and $100 \%$ (7/7). After sequencing, identical alleles were found in matched BF and TE samples. In summary, BF-DNA could be detected using MDA-WGA and PCR, and sequences in PCR positive samples were identical in matched BF-DNA and TE samples.
\end{abstract}

\section{Introduction}

Preimplantation genetic diagnosis (PGD) has become a widely accepted and routine technique for testing chromosomal integrity during in vitro fertilization (IVF) procedures, and has been successfully applied to high-risk couples for more than two decades [1,2]. During the development of PGD, polar bodies, blastomeres, and trophectoderms (TE) have been used as biopsy material for analysis. The polar body is expelled from oocytes during the first meiotic division, and therefore only contains maternal genetic information. Blastomere biopsies are typically performed on third day (D3) embryos that contain at least six cells, and one or two blastomeres are used for subsequent genetic analysis, including chromosome examination, identifying chromosomal structural abnormalities, molecular diagnosis of single gene diseases, and several other tests. The main disadvantage of these D3 biopsies of blastomeres is that any resultant analysis cannot completely rule out the impact of mosaics, affecting detection accuracy. Generally, TE biopsies are performed five days (D5) after fertilization, with three to ten trophoblast cells obtained for further genetic testing. This can improve the accuracy and validity of PGD [3-5]. Although these three PGD methods have achieved respectable results with embryos biopsied at either the zygote, cleavage, or blastocyst stages, zona drilling is required for each technique. This is accompanied by lesions to the embryo, the extent of which depends on the technique. However, it is not clear whether this damage affects the development of the embryo or fetus.

The blastocyst consists of three principal morphological components, the inner cell mass (ICM), the trophectoderm (TE), and the blastocoel fluid (BF). Although investigation of the ICM and TE have contributed to the understanding of embryogenesis and affected clinical IVF outcomes, any potential use of the BF has been largely overlooked. BF-DNA (deoxyribonucleic acid) has been identified in BFs. Those may be either free DNA or a particulate form, such as micro-particles. These are membrane-bound vesicles containing nuclear molecules that are released by membrane blebbing during cell death and replication [6,7]. Whichever form of BF-DNA takes, several studies have demonstrated that it has the potential to be used for PCR amplification and identifying mutations responsible for genetic diseases or chromosomal abnormalities. Despite this, the value of BF-DNA as a source of genetic material is controversial for use in PGS/PGD $[8,9]$. Compared to the traditional biopsy methods of blastomere and TE cell sampling, collecting material from the BF requires only that fluid is drawn out with an ICSI needle. This is less damaging to the embryo than other techniques. However, preliminary research demonstrates inconsistent and conflicting results, so further studies are needed to validate the approach and determine the value of BF-DNA in PGD.

Spinal muscular atrophy (SMA), phenylketonuria(PKU), and sex linked chromosomal disorders are all common genetic diseases observed in clinics. Our study focused on the detection and potential applications of genetic material found in human BF. We used diagnostic PCRs targeting, SMA, PKU, SRY and $\beta$-actin as biomarkers to evaluate whether these markers can be successfully amplified from human BF material after multiple displacement amplification (MDA) based whole gene amplification (WGA). Finally, we compared the consistency of sequencing from genetic material from the human $\mathrm{BF}$ and $\mathrm{TE}$.

Correspondence to: Wei He, Reproductive Medical Center, Department of Obstetrics and Gynecology, Southwest Hospital, Third Military Medical University, 30 Gaotanyan Street, Shapingba District, Chongqing 400038, China, Tel: 86-23-13608356955; Fax: 86-23-68754410; E-mail: anyhewei@163.com

Key words: blastocoel fluid, embryo biopsy, preimplantation genetic diagnosis, whole genome amplification, in vitro fertilization

Received: March 26, 2017; Accepted: April 10, 2017; Published: April 13, 2017 


\section{Materials and methods}

\section{Ethics statement}

This research was approved by the Ethics Committee (201684) at Southwest Hospital, Chongqing, China. All donors signed informed consent forms.

\section{Blastocyst culture}

Freshly donated D3 embryos were placed in $30 \mu \mathrm{L}$ droplets of G-2 Plus blastocyst medium (Vitrolife, Sweden) containing washed and pre-gassed mineral oil (Vitrolife, Sweden). These were cultured to the blastocyst stage under $6.5 \% \mathrm{CO}_{2}, 5 \% \mathrm{O}_{2}$, and balanced $\mathrm{N}_{2}$ at $37^{\circ} \mathrm{C}$ in Thermo 3131 incubators (Thermo, USA). After continuous culture for two days, the development and quality of the blastocysts were evaluated according to the blastocyst scoring system (Baczkowski et al., 2004). Blastocyst scores above IVBC or IVCB were selected for further BF collection.

\section{BF collection}

BF material was collected at the Reproductive Medical Center of Southwest Hospital (Chonqing, China) after approval of the local Ethics Committee. All BF aspiration was undertaken in an IVF laboratory following good laboratory practice guidelines [8], using methods for blastocyst micro-puncture and aspiration previously described [9]. BF samples were aspirated from expanded blastocysts using an ICSI pipette under the following specific steps. Firstly, the ICM was adjusted to the 12 o'clock position using a holding needle, and then the ICSI needle was inserted through a TE cell junction point at the 3 o'clock position. BF was drawn out slowly. Retrieved fluid was transferred to PCR tubes with $4-\mu \mathrm{l}$ cell lysis buffer (Cell Lysis Solution, 158908, QIAGEN) on ice and then centrifuged at $1000 \times g$ for $1 \mathrm{~min}$ for subsequent WGA analysis. The volume of BF was calculated according to a formula for the volume of a sphere: $V=4 \pi R^{3} / 3$, using a diameter of $200-220 \mu \mathrm{m}$. This was found to be the diameter range for an expanded blastocyst in this study.

\section{TE biopsy}

After BF collection, a 20-25 $\mu \mathrm{m}$ breach was opened at the ICSI pipet position by excising laser pulses. 2-3 hours later, TE cells herniated through this breach. After incubation, three to five TE cells biopsies were performed by excision using laser pulses. TE cells were transferred to PCR tubes with 4- $\mu$ l cell lysis buffer (Cell Lysis Solution, 158908, QIAGEN) on ice followed by centrifugation at $1000 \times g$ for $1 \mathrm{~min}$ to be used for subsequent WGA analysis.

\section{Whole genome amplification of BF and TE cells}

MDA-WGA of BF samples were performed using the REPLI- $\mathrm{g}^{\mathbb{R}}$ Single Cell kit (Qiagen), the detail protocol as follows Firstly, sufficient Buffer D2 (denaturation buffer) was prepared for the total number of whole genome amplification reactions; in this case, $3 \mu$ DTT $1 \mathrm{M}$ was added to $33 \mu \mathrm{l}$ buffer DLB for 12 reactions. Next, $3 \mu \mathrm{l}$ buffer D2 was added to the $4 \mu \mathrm{l}$ cell lysis buffer containing BF or TE on ice, and mixed carefully by flicking the tube and brief centrifugation. This mixture was incubated for $10 \mathrm{~min}$ at $65^{\circ} \mathrm{C}$, and then $3 \mu \mathrm{l}$ of stop solution was immediately added, mixed, and stored on ice. A master mix was prepared (each reaction required $29 \mu$ REPLI-g sc Reaction Buffer, 2 $\mu \mathrm{l}$ REPLI-g scDNA polymerase, and $9 \mu \mathrm{H}_{2} \mathrm{O}$ sc) and then $40 \mu \mathrm{l}$ of master mix was added to $10 \mu \mathrm{l}$ of each denatured DNA sample, mixed, and briefly centrifuged. This mixture was incubated at $30^{\circ} \mathrm{C}$ for $8 \mathrm{~h}$ and then inactivated by incubation at $65^{\circ} \mathrm{C}$ for $3 \mathrm{~min}$. Resultant reactions were checked using $1.5 \%$ agarose gel electrophoresis to verify the WGA products.

\section{Primer design}

Specific primers for SMA, PKU, SRY and $\beta$-actin amplification were designed using Primer 5.0 software. Sequence specificity was confirmed using BLAST analysis software (http://blast.ncbi.nlm.nih. gov/) against the human genomic plus transcript database. Primers were synthesized by Bioligo Biotech (Shanghai, China), and the primer details were shown in Table 1.

\section{Amplification and detection of genes by PCR and sequencing method}

The PCR protocol used the following conditions: initial denaturation at $94^{\circ} \mathrm{C}$ for $5 \mathrm{~min}$ and then 35 cycles of denaturation at $94^{\circ} \mathrm{C}$ for $35 \mathrm{~s}$, annealing at $55^{\circ} \mathrm{C}$ for $35 \mathrm{~s}$, and extension at $72^{\circ} \mathrm{C}$ for $1 \mathrm{~min}$. The final elongation was taken for $5 \mathrm{~min}$ at $72^{\circ} \mathrm{C} .1 .5 \%$ agarose electrophoresis was used for the detection of PCR products, and visualized using UV (Biorad GelDoc XR, USA).

Specific PCR products were isolated and purified using agarose gel electrophoresis DNA Recovery Kit (TIANgel Midi Purification Kit), and sent for sequencing at BGI (Shanghai, China). All sequencing results were analyzed using DNAstar analysis software to compare homology between samples and with the NCBI database.

\section{Results}

Seven donated blastocyst were used in this study, the volume range for the BF of approximately 0.001-0.002 $\mu$ l.

\section{WGA of blastocoel fluid compared TE cell WGA}

All samples were amplified using MDA-WGA and then visualized on a $1.5 \%$ agarose gel. This revealed that the pattern of DNA bands after WGA was similar between BF and TE cells from the same blastocyst, with most product bands ranging from $100 \mathrm{bp}$ to $1000 \mathrm{bp}$ (Figure 1).

\section{Target amplification from blastocoel fluid using PCR}

Seven TE cell samples were amplified using primers specific for SMA, PKU, SRY and $\beta$-actin. Positive bands were observed for each, except for amplification of the SRY primers in two samples (TE 1 and 2) (Figure 2, TE group). However, of the seven $B F$ samples, three were positive for SMA $(42.9 \%, 3 / 7)$, two for PKU $(28.6 \%, 2 / 7)$, three for SRY amplification $(60 \%, 3 / 5)$, and five were positive using primers for $\beta$-actin $(71.4 \%, 5 / 7)$.

\section{Sequencing of target genes}

Sequencing analysis revealed that the SMA, PKU, SRY and $\beta$-actin regions from the TE group were completely consistent (Table 2 ). When comparing the sequencing of BF samples with positive TE samples, results were identical between sample type (Table 2). There were identical comparing the positive productions between $\mathrm{BF}$ and $\mathrm{TE}$ in the same blastocyst.

\section{Discussion}

Preimplantation genetic diagnosis (PGD) was first reported in England in 1990 and was a considerable advancement in the reproductive medicine $[7,8]$. Polar bodies, cleavage balls, and TE cells have all been used as biopsy materials during the development of PGD. However, these biopsy methods introduce unavoidable risk to the embryo and its further development [9]. Blastocoel fluid (BF) biopsies 


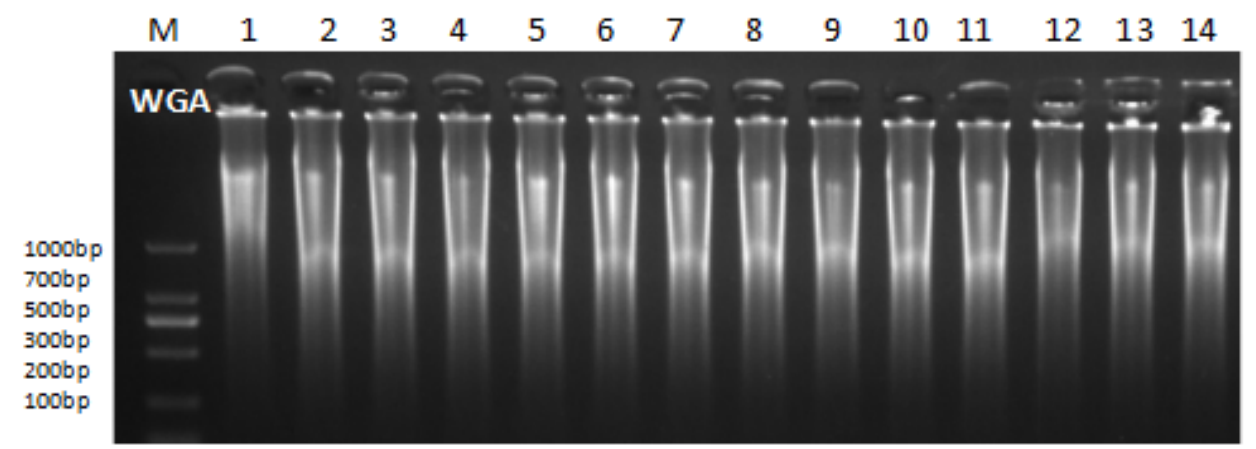

Figure 1. WGA products of TE and BF on agarose gel. M: DNA marker No 1-7: WGA products of BF material, No 8-14: WGA products of TE material.

A

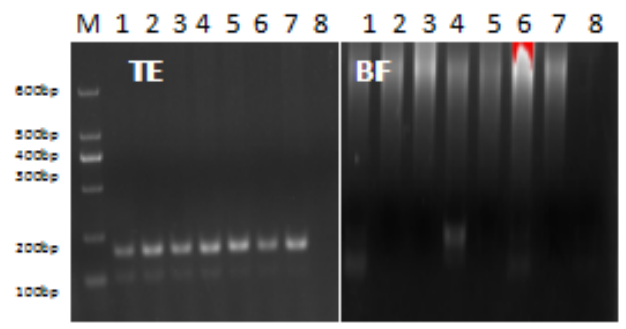

C

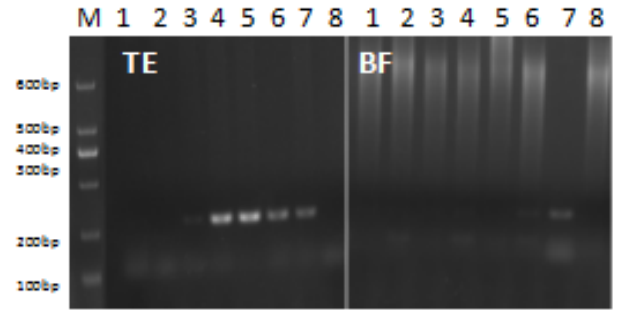

B

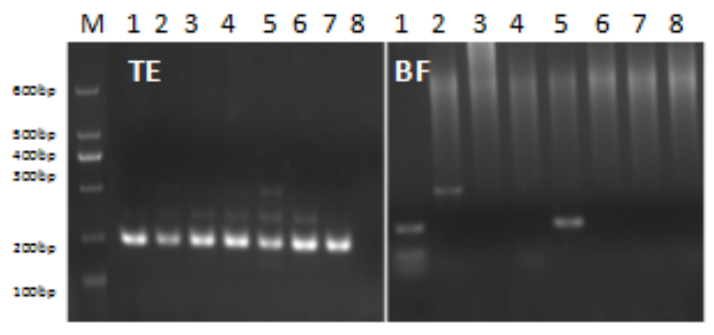

D

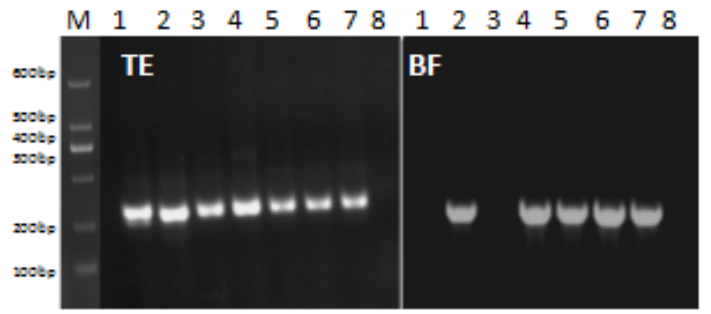

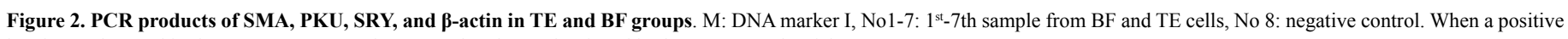
band was observed in the BF group, a complementary band was also found in the same sample of the TE group.

Table 1. The specific primers for SMA, PKU, SRY and $\beta$-actin amplification after the WGA.

\begin{tabular}{|c|l|c|}
\hline Name & \multicolumn{1}{|c|}{ Sequence (5)-3 $\mathbf{~})$} & Length (bp) \\
\cline { 1 - 2 } SMA F & GGCATAGAGCAGCACTAAATGAC & \multirow{2}{*}{188} \\
\cline { 1 - 2 } SMA R & CATTGCATTTGGTTATTACATAAC & \multirow{2}{*}{266} \\
\hline PKU F & GAACTTTGCTGCCACAATACCTCGGC & \\
\hline PKU R & CAAGAAAGTTGGGTACTTTATTTTCT & \multirow{2}{*}{286} \\
\hline SRY F & GATGACTGTACGAAAGCCACACA & \\
\hline SRY R & AAATGAATAAGGCCTTAG & \\
\hline$\beta$-actin F & CTCCATCCTGGCCTCGCTGT & \\
\hline$\beta$-actin R & GCTGTCACCTTCACCGTTCC & \\
\hline
\end{tabular}

are much safer and easier to get for the simply drawing with an ICSI needle.

Studies within other settings have found that it is possible to obtain BF-DNA, using RT-PCR to analyze several targeted genes (GADPH, TSPY1, and TBC1D3) A whole-genome microarray was also attempted on five amplified DNA products obtained from the BF, but found a karyotype in only two samples [10]. Polisseni et al. reported on the use of DNA fingerprinting for eight chromosomes from 11 paired BFDNA samples separated from whole embryo DNA [11]. This was used to determine the concordance of polymorphic loci between paired samples, showing that $100 \%$ of the loci successfully amplified using samples from the whole embryo, but DNA from the BF was of inferior quality and unable to provide a complete DNA fingerprinting analysis. Genomic DNA from the BF can be amplified and characterized by comprehensive chromosome microarrays. However, the high discordance between karyotypes obtained from BF-DNA and ICM-TE suggested that using $\mathrm{BF}$ genetic material for preimplantation genetic testing was not ideal.

On the contrary, Magli et al. (2016) recently demonstrated a successful attempt to investigate the potential of BF. In their study, they analyzed BF that had been extracted from differentiated blastocysts, and compared the ploidy status of BF-DNA to polar bodies, a single blastomere, or TE cells $[12,13]$. They identified a total ploidy concordance of $94.9 \%$ between BF-DNA and matched polar body or blastomere cells. Additionally, they identified a $97.4 \%$ ploidy concordance between BF-DNA and TE cells. Palini et al. (2015) showed that genomic DNA was present in approximately $90 \%$ of blastocoel fluid samples harvested during vitrification procedures [14].

In our study, we successfully extracted BF and confirmed the presence of DNA fragments. These were analogous WGA patterns between the BF and TE from the same blastocyst after amplified by MDA-WGA. However, the positive results for the detecting biomarkers was disappointing with low amplification efficiency. Positive PCR for SMA, PKU, and SRY regions were $42.9 \%$ (3/7), 60\% (3/5), and $42.9 \%$ (3/7) respectively in BF, but $100 \%$ in TE samples except the SRY, it 
Table 2. Alignment results of SMA, PKU, SRY and $\beta$-actin among TE, BF and template gene in Genebank database.

\begin{tabular}{|c|c|c|c|c|c|c|c|c|c|c|c|c|c|c|}
\hline $\begin{array}{l}\text { sample } \\
\text { gene }\end{array}$ & 1-TE & $1-\mathrm{BF}$ & 2-TE & 2-BF & 3-TE & 3-BF & 4-TE & 4-BF & 5-TE & $5-\mathrm{BF}$ & 6-TE & 6-BF & 7-TE & $7-\mathrm{BF}$ \\
\hline $\begin{array}{c}\text { SMA } \\
\text { (GI:KU524731.1) }\end{array}$ & + & ++ & + & & + & & + & ++ & + & & + & ++ & + & \\
\hline $\begin{array}{c}\text { PKU } \\
\text { (GI:KR711257.1) }\end{array}$ & + & ++ & + & & + & & + & & + & ++ & + & & + & \\
\hline $\begin{array}{c}\text { SRY } \\
\text { (GI:NM_003140.2) }\end{array}$ & + & & + & & + & & + & ++ & + & & + & ++ & + & ++ \\
\hline $\begin{array}{c}\beta \text {-actin } \\
\text { (GI:NG_023870.2) }\end{array}$ & + & & + & & + & ++ & + & ++ & + & ++ & + & ++ & + & ++ \\
\hline
\end{tabular}

+: The consistency between TE and template gene

++ : The consistency between TE and BF.

is possible that there was no $\mathrm{Y}$ chromosome in these samples. It was shown that BF-DNA is unsuitable for single gene detection. However, sequencing of PCR products revealed a high similarity between BF and TE samples. A reason for the low efficiency may be the very low BF volumes that were extracted (0.001-0.002 $\mu \mathrm{l})$ or the insufficient for the WGA technology. The high similarity shown that when the efficiency of WGA was increased, BF can be used to determine the genetic condition of the embryo.

Multiple annealing and looping-based amplification cycles (MALBAC) is a quasilinear whole genome amplification method. Unlike conventional DNA amplification methods that are non-linear or exponential (in each cycle, DNA copied can serve as template for subsequent cycles), MALBAC utilizes special primers that allow amplicons to have complementary ends and therefore to loop, preventing DNA from being copied exponentially. This results in amplification of only the original genomic DNA and therefore reduces amplification bias [17]. A previous study has shown that MALBAC has better genome recovery sensitivity and lower allele dropout compared to MDA [18] and the requirement of DNA is only $0.5 \mathrm{pg}$. We speculate that this method may increase the positive rate of amplification from BF-DNA and more investigation is required. It may be more suitable than MDA in the micro-amount materials analysis of WGA amplification.

In conclusion, the BF volume of a blastocyst was ranged from 0.001-0.002 $\mu \mathrm{l}$ and blastocoel fluid harvested during the verification process possesses amplifiable DNA fragments suitable for WGA and PCR. The specific PCR results shown high similarity between human $\mathrm{BF}$ and all TE from the samples. Although low amplification efficiency was found using MDA-WGA, it could be modified with other method, such as MALBAC. BF should be a potential source for PGD/PGS in the future.

\section{Acknowledgements}

This study was supported by grants from the National Sciencetechnology Support Plan Projects (2012BAI31B). The authors would like to thank each of the families that participated in the study and are grateful to the Third Military Medical University Institute of Immunology for providing experimental instruments and equipment.

\section{Conflict of interest}

All authors declared that they have no conflicts of interest to this work. We declare that we do not have any commercial or associative interest that represents a conflict of interest in connection with the work submitted.

\section{References}

1. Keltz MD, Vega M, Sirota I, Lederman M, Moshier EL, et al. (2013) Preimplantation genetic screening (PGS) with Comparative genomic hybridization (CGH) following day 3 single cell blastomere biopsy markedly improves IVF outcomes while lowering multiple pregnancies and miscarriages. J Assist Reprod Genet 30: 1333-1339.

2. Zakharova EE, Zaletova VV, Krivokharchenko AS (2014) Biopsy of human morulastage embryos: outcome of 215 IVF/ICSI cycles with PGS. PLoS One 9: e106433. [Crossref]

3. Cimadomo D, Capalbo A, Ubaldi FM, Scarica C, Palagiano A, et al. (2016) The Impact of Biopsy on Human Embryo Developmental Potential during Preimplantation Genetic Diagnosis. Biomed Res Int 2016: 7193075.

4. Palini S, De Stefani S, Primiterra M, Galluzzi L (2015) Pre-implantation genetic diagnosis and screening: now and the future. Gynecol Endocrinol 31: 755-759.

5. Harton G, Braude P, Lashwood A, Schmutzler A, Traeger-Synodinos J, et al. (2011) ESHRE PGD consortium best practice guidelines for organization of a PGD centre for PGD/preimplantation genetic screening. Hum Reprod 26: 14-24. [Crossref]

6. Harton GL, De Rycke M, Fiorentino F, Moutou C, SenGupta S, et al. (2011) ESHRE PGD consortium best practice guidelines for amplification-based PGD. Hum Reprod 26: 33-40. [Crossref]

7. Thornhill AR, deDie-Smulders CE, Geraedts JP, Harper JC, Harton GL, et al (2005) ESHRE PGD Consortium 'Best practice guidelines for clinical preimplantation genetic diagnosis (PGD) and preimplantation genetic screening (PGS). Hum Reprod 20: 35-48.

8. Magli MC, Pomante A, Cafueri G, Valerio M, Crippa A, et al. (2016) Preimplantation genetic testing: polar bodies, blastomeres, trophectoderm cells, or blastocoelic fluid? Fertil Steril 105: 676-683.

9. Hardy K, Martin KL, Leese HJ, Winston RM, Handyside AH (1990) Human preimplantation development in vitro is not adversely affected by biopsy at the 8-cell stage. Hum Reprod 5: 708-714.

10. Lu L, Lv B, Huang K, Xue Z, Zhu X, et al. (2016) Recent advances in preimplantation genetic diagnosis and screening. J Assist Reprod Genet 33: 1129-1134. [Crossref]

11. Polisseni J, Sa WF, Guerra Mde O, Machado MA, Serapiao RV, et al. (2010) Postbiopsy bovine embryo viability and whole genome amplification in preimplantation genetic diagnosis. Fertil Steril 93: 783-788.

12. Handyside AH, Kontogianni EH, Hardy K, Winston RM (1990) Pregnancies from biopsied human preimplantation embryos sexed by Y-specific DNA amplification. Nature 344: 768-770. [Crossref]

13. Pinborg A, Ortoft G, Loft A, Rasmussen SC, Ingerslev HJ (2015) Cervical conization doubles the risk of preterm and very preterm birth in assisted reproductive technology twin pregnancies. Hum Reprod 30: 197-204.

14. Jensen PL, Beck HC, Petersen J, Hreinsson J, Wånggren K, et al. (2013) Proteomic analysis of human blastocoel fluid and blastocyst cells. Stem Cells Dev 22: 1126-1135. [Crossref]

15. Dardik A, Schultz RM (1991) Protein secretion by the mouse blastocyst: differences in the polypeptide composition secreted into the blastocoel and medium. Biol Reprod 45: 328-333.

16. Xu J, Fang R, Chen L, Chen D, Xiao JP, et al. (2016) Non-invasive chromosome screening of human embryos by genome sequencing of embryo culture medium for in vitro fertilization. Proc Natl Acad Sci U S A 113: 11907-11912. 
17. Zong C, Lu S, Chapman AR, Xie XS (2012) Genome-wide detection of singlenucleotide and copy-number variations of a single human cell. Science 338: 1622-1626. [Crossref]
18. Hou Y, Wu K, Shi X, Li F, Song L, et al. (2015) Comparison of variations detection between whole-genome amplification methods used in single-cell resequencing. GigaScience 4: 37. [Crossref]

Copyright: (C2017 Shangguan T. This is an open-access article distributed under the terms of the Creative Commons Attribution License, which permits unrestricted use, distribution, and reproduction in any medium, provided the original author and source are credited. 